\title{
Fully differential cross sections for the single ionization of helium by fast ions: Classical model calculations
}

\author{
L. Sarkadi* \\ Institute for Nuclear Research of the Hungarian Academy of Sciences (ATOMKI), H-4001 Debrecen, Pf. 51, Hungary
}

(Received 5 March 2018; published 16 April 2018)

\begin{abstract}
Fully differential cross sections (FDCSs) have been calculated for the single ionization of helium by 1- and $3-\mathrm{MeV}$ proton and $100-\mathrm{MeV} / \mathrm{u} \mathrm{C}^{6+}$ ion impact using the classical trajectory Monte Carlo (CTMC) method in the nonrelativistic, three-body approximation. The calculations were made employing a Wigner-type model in which the quantum-mechanical position distribution of the electron is approximated by a weighted integral of the microcanonical distribution over a range of the binding energy of the electron. In the scattering plane, the model satisfactorily reproduces the observed shape of the binary peak. In the region of the peak the calculated FDCSs agree well with the results of continuum-distorted-wave calculations for all the investigated collisions. For 1-MeV proton impact the experimentally observed shift of the binary peak with respect to the first Born approximation is compared with the shifts obtained by different higher-order quantum-mechanical theories and the present CTMC method. The best result was achieved by CTMC, but still a large part of the shift remained unexplained. Furthermore, it was found that the classical theory failed to reproduce the shape of the recoil peak observed in the experiments, it predicts a much narrower peak. This indicates that the formation of the recoil peak is dominated by quantum-mechanical effects. For $100-\mathrm{MeV} / \mathrm{u} \mathrm{C}^{6+}$ ion impact the present $\mathrm{CTMC}$ calculations confirmed the existence of the "double-peak" structure of the angular distribution of the electron in the plane perpendicular to the momentum transfer, in accordance with the observation, the prediction of an incoherent semiclassical model, and previous CTMC results. This finding together with wave-packet calculations suggests that the " $\mathrm{C}^{6+}$ puzzle" may be solved by considering the loss of the projectile coherence. Experiments to be conducted using ion beams of anisotropic coherence are proposed for a more differential investigation of the ionization dynamics.
\end{abstract}

DOI: 10.1103/PhysRevA.97.042703

\section{INTRODUCTION}

The single ionization of atoms by charged particle impact is a three-body breakup problem that represents a serious challenge from a theoretical point of view due to the long-range Coulomb force acting between the particles. The most complete information about the breakup process can be obtained by measuring the fully differential cross section (FDCS) for the investigated collision system. Despite the fundamental importance of hydrogen as target atom, due to the well-known difficulties of preparing an atomic hydrogen target, most of the FDCS measurements were made on heavier atoms, primarily on helium.

FDCSs are obtained in kinematically complete experiments. In the field of atomic collisions a very efficient technique, namely the COLTRIMS (cold-target-recoil-ionmomentum spectroscopy) [1] has been developed for this purpose. In past decades COLTRIMS has been applied in many investigations and went through a remarkable progress. Its power has been demonstrated by a recent experiment carried out by Gassert et al. [2] in which FDCS for 1-MeV protons on helium collisions was measured with unprecedented momentum resolution. The aim of this accurate experiment was to shed light on the possible sources of the disagreement between theory and previous experiments, as well as to provide benchmark data for future FDCS investigations. 1-MeV

\footnotetext{
*sarkadil@atomki.hu
}

protons have a small perturbation strength $\left(Z_{p} / v=0.16\right)$; therefore, the measured FDCSs are expected to be reproduced reasonably well even by the simplest perturbation theory, the first Born approximation (FBA).

In the experiment in Ref. [2] the three-dimensional emission pattern of the electron ejected with energy of $6.5 \mathrm{eV}$ was measured. The momentum transfer $q$ was fixed at 0.75 a.u. The obtained data clearly show the theoretically expected double-lobe structure consisting of the so-called binary and recoil peak. The binary peak is attributed to electrons ejected approximately in the direction of $\boldsymbol{q}$ as a result of a direct, binary collision with the projectile. The recoil peak occurs in the vicinity of the $-\boldsymbol{q}$ direction and it is attributed to electrons backscattered of the target nucleus. The ionization theories (e.g., FBA) predict a distinct node between the two peaks. The benchmark experiment in Ref. [2] proved the existence of the node. This is a remarkable result, because previous experiments [3-5] did not show the node in strong disagreement even with the most advanced theories. Although the failure of the latter experiments to observe the node most likely was due to the insufficient momentum resolution, other instrumental effects cannot be ruled out. Furthermore, it should be noted that the previous FDCS measurements for helium were made at different impact energies and with different projectile ions; therefore, they cannot be compared directly with the measurement in Ref. [2].

To compare their experimental data to the theory, the authors of Ref. [2] performed calculations applying two theoretical descriptions. One was the plane-wave first Born approximation 
(PWFBA). Several versions of PWFBA were considered, differing in the choice of the electronic wave function in the initial and final state. The other description was the eikonalwave Born approximation (EWBA), which is a variant of the continuum distorted wave (CDW) approach. Since no absolute measurements were made in Ref. [2], only the shape of the angular distribution was compared with the theoretical predictions. Excellent agreement was found except for a small, but definite deviation between the measured and calculated position of the binary peak. In the scattering plane, defined by the initial and final momentum vector of the projectile $\left(\boldsymbol{p}_{i}\right.$ and $\boldsymbol{p}_{f}$, respectively), the experimental peak position is $\theta_{e}=73^{\circ}$ (here $\theta_{e}$ is the polar angle of the ejected electron in a coordinate system where the $z$ axis is taken parallel with $\boldsymbol{p}_{i}$ ). All the PWFBA calculations yielded $\theta_{e}=79^{\circ}$, a value one expects assuming that the electron is emitted exactly in the direction of the momentum transfer, $\boldsymbol{q}=\boldsymbol{p}_{i}-\boldsymbol{p}_{f}$.

The shift of the observed binary peak towards smaller angles indicated the presence of higher-order effects in the electron emission. Indeed, the EWBA calculations partly accounted for the shift, resulting in a peak position that was lower by about $3^{\circ}$ than that predicted by PWFBA. This finding was confirmed also by an estimate obtained from a second-order Born calculation.

The experimental results of Ref. [2] have motivated further theoretical works. Voitkiv [7] has made calculations also in FBA and considered two distorted-wave approaches: the continuum-distorted-wave eikonal-initial-state (CDW-EIS) and the symmetric eikonal (SEA) models. His procedure was based on calculation of impact-parameter dependent transition amplitudes in the framework of the semiclassical approximation. This was made in the three-body approximation using an effective target potential [8]. $\boldsymbol{q}$-dependent quantum mechanical transition amplitudes were obtained from the semiclassical ones by a suitable Fourier transformation. From the comparison of the obtained results with the experimental data Voitkiv drew similar conclusions as the authors of Ref. [2]: although the CDW models considerably improved the agreement between theory and experiment as compared to FBA, a shift of about $5^{\circ}-6^{\circ}$ still remained between the calculated and observed position of the binary peak. The shapes of the angular distributions in both the scattering and azimuthal plane were reproduced well by the CDW calculations. A small discrepancy was found between theory and experiment for the recoil-to-binary-peak ratio: the theory overestimated it by $25 \%$.

Chuluunbaatar et al. [9] performed FDCS calculations beyond PWFBA in order to clarify the role of higher-order collision mechanisms. For this purpose they used the planewave second Born approximation (PWSBA) and the 3C approaches. In the 3C (or BBK) model, proposed by Brauner et al. [10], three Coulomb continuum functions are applied for the description of the final-state interactions. The authors repeated the calculations using three different ground-state trial wave functions of helium, characterized by different degrees of electron correlation. Similar to the previous theoretical efforts, the models considered by Chuluunbaatar et al. gave only a partial account of the shift of the binary peak. At the same time, concerning the recoil-to-binary-peak ratio a good agreement with the experiment was achieved by the $3 \mathrm{C}$ model applying strongly correlated ground-state helium wave function.
In this work we investigated the performance of the classical trajectory Monte Carlo (CTMC) method in the calculation of FDCS by comparing the results obtained by the method with the benchmark experimental data of Ref. [2]. Although CTMC has been widely used for the description of ion-atom collision processes, its application in FDCS studies is rather scarce [11-18]. The advantage of CTMC is that it is a nonperturbative theory that provides an exact description of the full dynamics of the three-body breakup process, albeit classically. It was an interesting question whether CTMC could resolve the remaining discrepancy between the experiment and the higherorder quantum-mechanical theories found for the angular shift of the binary peak with respect to FBA.

The theoretical calculation of FDCS, as the most differential quantity, strongly depends on the applied approximations. In FDCS studies of the ionization of atoms by electron impact it turned out that the standard CTMC formulated by Abrines and Persival [19] failed to reproduce the binary peak $[20,21]$. The failure of the theory was traced back to the poor approximation of CTMC to mimic the quantum-mechanical position distribution of the electron in the initial state. The method is based on the probability density of the classical microcanonical ensemble of the electronic states in the phase space. While this results in a proper momentum distribution, the exclusion of the spatial region of negative kinetic energies in the classical theory leads to a position distribution that strongly deviates from the quantum-mechanical one. Several attempts were made to overcome this handicap of CTMC [2225]. The derived phase-space probability density functions can be regarded as approximations of the Wigner function [26]; therefore, the corresponding CTMC approaches may be referred to as "CTMC based on Wigner distribution," while the standard theory is called simply "microcanonical CTMC."

In the present work we improved the procedure suggested by Hardie and Olson [23,25]. These authors approximated the quantum-mechanical initial radial position distribution by a discrete superposition of microcanonical ensembles over a suitably chosen range of the binding energy of the electron. Instead of discrete superposition we considered a continuous sum, a weighted integral over the binding energy. We carried out the FDCS calculations with initial position distributions derived from different trial ground-state wave functions of helium, similar to the quantum-mechanical investigations. Besides the case of $1-\mathrm{MeV}$ proton impact, in the paper we present also results obtained with the improved method for $3-\mathrm{MeV}$ protons and $100-\mathrm{MeV} / \mathrm{amu} \mathrm{C}^{6+}$ ions, and compare them with measured data $[3,6]$.

Atomic units are used throughout the paper unless otherwise stated.

\section{THEORY}

The CTMC method is well documented in the literature, so we summarize it only briefly here. We applied the threedimensional, nonrelativistic three-body version of the theory including all the interactions between the particles: the projectile ion, the atomic electron, and the target core. The interactions with the target core are approximated by either the Coulomb potential of an effective nucleus charge, or by a model potential. For the latter we used the Green-Sellin-Zachor 
(GSZ) potential [27] based on Hartree-Fock calculations. It has the following form for a neutral atom:

$$
V(r)=-[(Z-1) \Omega(r)+1] / r,
$$

where $Z$ is the nuclear charge and

$$
\Omega(r)=\{(\eta / \xi)[\exp (\xi r)-1]+1\}^{-1} .
$$

The values of $\eta$ and $\xi$ were taken from Garvey et al. [28]. For helium $\eta=1.77$ and $\xi=2.625$.

The dynamical properties of the collision system are determined from the statistical analysis of a large number of particle trajectories obtained from the numerical solution of Newton's equations of motion under randomly chosen initial conditions (impact parameter and five further parameters defining the position and velocity vector of the atomic electron).

The details of the used CTMC computer code are given in [29]. The integration of the equations of motion was started at a distance of several hundreds of atomic units from the target, where the projectile exerted negligible perturbation on the electron. In the outgoing phase, for a collision leading to ionization the trajectories of the particles were calculated up to $10^{8}$ a.u. in order to determine the postcollisional effects $[30,31]$ accurately.

For large number $N_{c}$ of collision events characterized by uniformly distributed impact parameter $(B)$ values in the range $\left(0, B_{\max }\right)$, the FDCS for ejection of the electron with energy between $E_{e}$ and $E_{e}+d E_{e}$ into solid angle $d \Omega_{e}$, and for scattering of the projectile into solid angle $d \Omega_{p}$ is obtained as [18]

$$
\frac{d^{5} \sigma}{d E_{e} d \Omega_{e} d \Omega_{p}} \approx \frac{B_{\max } \sum_{j=1}^{N_{i}} B_{j}^{(i)}}{N_{c} \Delta E_{e} \Delta \Omega_{e} \Delta \Omega_{p}} .
$$

Here $N_{i}$ is the number of the collision events in which the electron is emitted into energy and solid angle window $\Delta E_{e}$ and $\Delta \Omega_{e}$, and the projectile is scattered into solid angle window $\Delta \Omega_{p} . B_{j}^{(i)}$, s are the impact parameters belonging to those collisions. The solid angles $\Delta \Omega_{k}(k=e, p)$ are determined by the minimum and maximum values of the respective polar and azimuthal angles, $\theta_{k}$ and $\phi_{k}$ :

$$
\begin{aligned}
\Delta \Omega_{k} & =\int_{\theta_{k}^{\min }}^{\theta_{k}^{\max }} \int_{\phi_{k}^{\min }}^{\phi_{k}^{\max }} \sin \theta_{k} d \theta_{k} d \phi_{k} \\
& =\left(\cos \theta_{k}^{\min }-\cos \theta_{k}^{\max }\right)\left(\phi_{k}^{\max }-\phi_{k}^{\min }\right) .
\end{aligned}
$$

The standard error of FDCS is [19]

$$
\frac{d^{5} \sigma}{d E_{e} d \Omega_{e} d \Omega_{p}}\left(\frac{N_{c}-N_{i}}{N_{c} N_{i}}\right)^{1 / 2} .
$$

We note here that for the calculation of FDCS with acceptable statistical error one has to integrate the equations of motion for a very large number of collisions. In the present work we followed the history of altogether $3.4 \times 10^{9}$ collision events.

We made a series of FDCS calculations differing in the choice of the initial position probability distribution of the electron. In the standard, microcanonical CTMC calculation we applied the general procedure suggested by Reinhold and Falcón [32] for non-Coulombic systems which is equivalent to the original Abrines and Percival's method [19] in the case

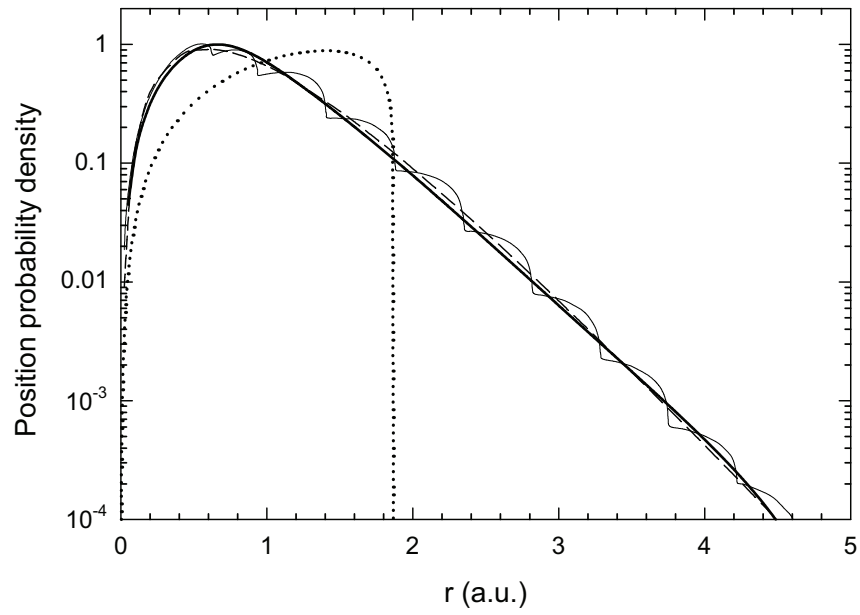

FIG. 1. Position distribution of the electron in the helium atom calculated by the present method (thick solid line) and by the procedure proposed in Refs. [23,25] (thin solid line) using hydrogenlike (single- $\zeta$ ) ground-state trial wave function. The dashed line represents the quantum-mechanical result. The dotted curve shows the microcanonical distribution used in the standard CTMC.

of the Coulomb interaction. In this model the radial position distribution is given by

$$
\rho_{\mathrm{MC}}\left(r, E_{b}\right)=N r^{2} \sqrt{2 \mu\left[E_{b}-V(r)\right]},
$$

where $N$ is a normalization constant, $\mu$ is the reduced mass of the electron in helium, and $E_{b}<0$ is the binding energy. The notation $\mathrm{MC}$ in $\rho_{\mathrm{MC}}\left(r, E_{b}\right)$ refers to "microcanonical."

In our present, extended Wigner-type CTMC model the quantum-mechanical position distribution $\rho_{\mathrm{QM}}(r)$ is approximated by a weighted integral of the microcanonical distribution given by Eq. (4) over the binding energy:

$$
\rho_{\mathrm{QM}}(r) \approx \int_{u_{\min }}^{u_{\max }} d u w(u) \rho_{\mathrm{MC}}(r,-u),
$$

with $u=-E_{b}$ (now $E_{b}$ is considered as a continuous variable of the model). This is a generalization of the procedure proposed by Hardie and Olson [23,25], who expressed the position distribution as a finite weighted sum of microcanonical distributions:

$$
\rho(r)=\sum_{i} w_{i} \rho_{\mathrm{MC}}\left(r, E_{b i}\right) .
$$

In Eq. (5) we chose the $w(u)$ function and the value of $u_{\min }$ and $u_{\max }$ in a way to achieve the best fit to $\rho_{\mathrm{QM}}(r)$ derived from a given ground-state wave function of helium.

To show the difference between the two procedures, in Fig. 1 we plotted the results obtained by Eqs. (5) and (6) for the case of hydrogenlike (single- $\zeta$ ) ground-state trial wave function of helium. The fit in this example was made to $\rho_{\mathrm{QM}}(r)$ using $V(r)=-Z_{\text {eff }} / r$ in Eq. (4) with $Z_{\text {eff }}=2-5 / 16$. Obviously, our procedure provided a better fit. In Fig. 1 we plotted also the microcanonical position distribution used in the standard CTMC. The latter shows a cutoff at $r=Z_{\text {eff }} /\left|E_{b}\right|=1.87$ a.u. in strong disagreement with the quantum distribution.

We considered further two, more realistic ground-state wave functions of helium. In both cases we used the GSZ potential 

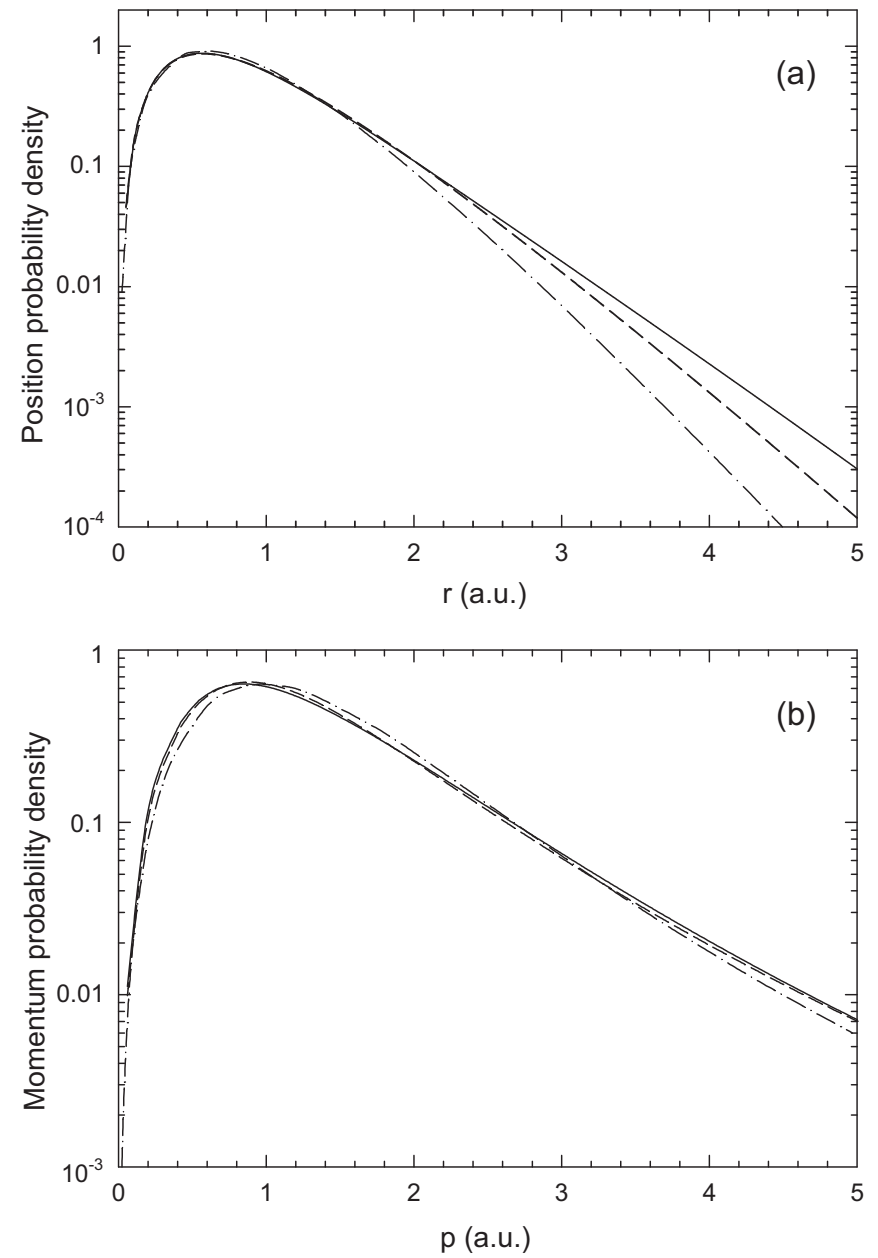

FIG. 2. Quantum-mechanical position (a) and momentum (b) distribution of the electron in the helium atom corresponding to single- $\zeta$ (dashed-dotted line), RHF [33] (dashed line), and SPM [34] (solid line) wave function.

for the calculation of $\rho_{\mathrm{MC}}(r,-u)$ in the integral (5). One was the Roothaan-Hartree-Fock (RHF) function [33]; the other was the Silverman-Platas-Matsen (SPM) function [34]. The position and momentum distributions, as well as the optimized $w(u)$ weight functions for the three trial wave functions, are summarized in the Appendix. In Fig. 2 we compare the three quantum-mechanical distributions. While for the position they greatly differ from each other at large distances, for the momentum the three distributions almost coincide.

The calculations in the extended CTMC model were made in the following way. At a given trial wave function for each collision event we selected randomly a $u$ value with a probability according to the corresponding $w(u)$ weight function. Taking $E_{b}=-u$, we selected randomly the initial parameters of the collision as prescribed in the standard, microcanonical CTMC, and solved the equations of motion. The FDCS obtained in this way for a series of collisions events with different $u$ values is a weighted average of microcanonical CTMC results.

As an example, in Fig. 3 we plotted the initial position and momentum distributions generated by our extended CTMC program for the SPM trial function. The distributions were cre-
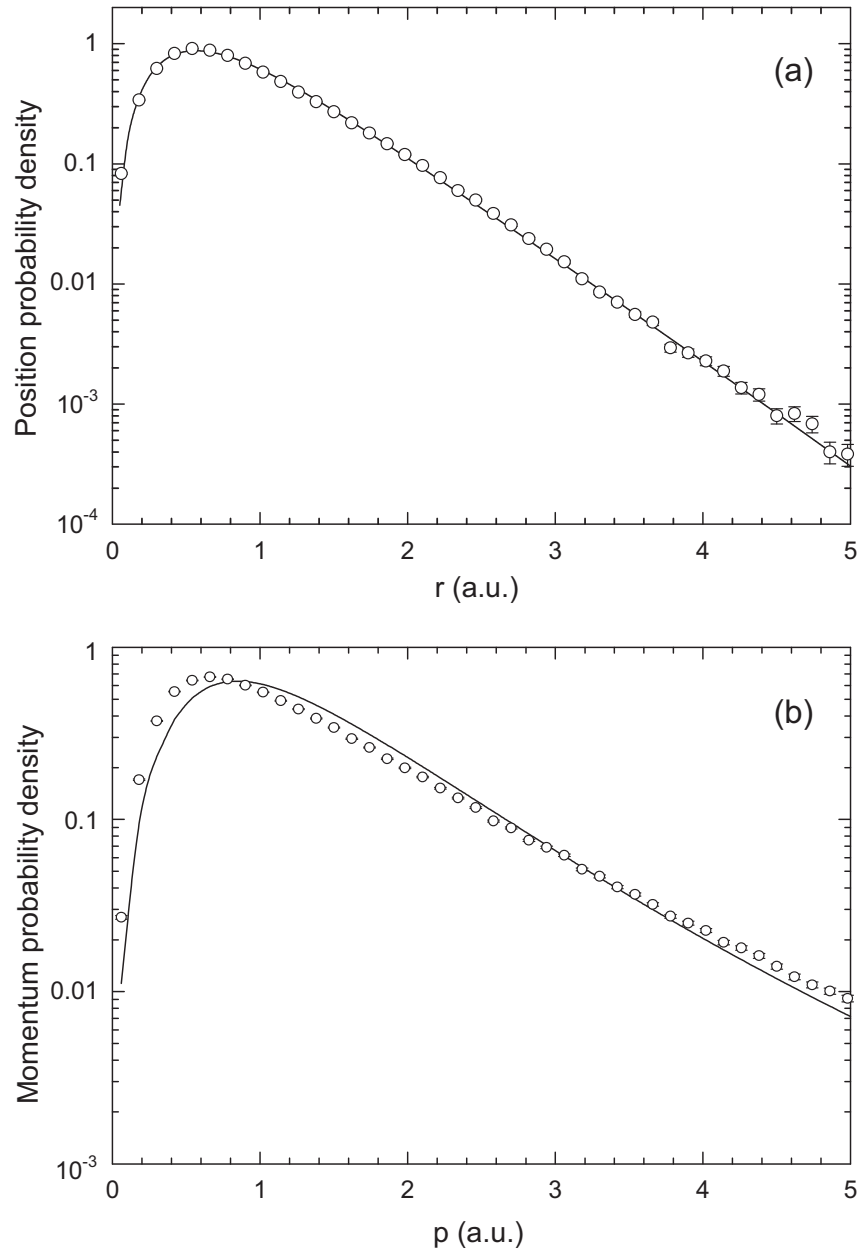

FIG. 3. Initial position (a) and momentum (b) distributions generated by the present extended CTMC program on the basis of Eqs. (4) and (5) for the SPM trial function (open circles). The solid lines represent the quantum-mechanical distributions.

ated considering $5 \times 10^{5}$ events. For the position the calculated data are in excellent agreement with the quantum distribution $\rho_{\mathrm{SPM}}(r)$. For the momentum the data slightly deviate from $\rho_{\mathrm{SPM}}(p)$.

\section{RESULTS AND DISCUSSION}

For $1 \mathrm{MeV}$-proton impact we made the calculations under conditions identical with those of the experiment [2], i.e., we considered in Eq. (2) collision events characterized by momentum transfer values $q=0.75 \pm 0.25$ a.u. and electron energies $E_{e}=6.5 \pm 3.5 \mathrm{eV}$. Furthermore, with the choice of the $x z$ plane for the scattering plane (see, e.g., Fig. 2 in Ref. [3]), the electron emission in the latter plane was restricted allowing deviation within $\Delta \phi_{e}= \pm 5^{\circ}$. One can easily show (see, e.g., Ref. [2]) that at $q=0.75$ a.u. and $E_{e}=6.5 \mathrm{eV}$ the scattering angle of the proton $\theta_{p}=0.063 \mathrm{mrad}$ and the polar angle of the momentum transfer vector $\theta_{\boldsymbol{q}}=76.1^{\circ}$. Figure 4 presents our FDCS results as a function of the polar angle of the electron obtained with different approximations. The calculation using the traditional microcanonical CTMC demonstrated the failure of the model in reproduction of the observed angular 


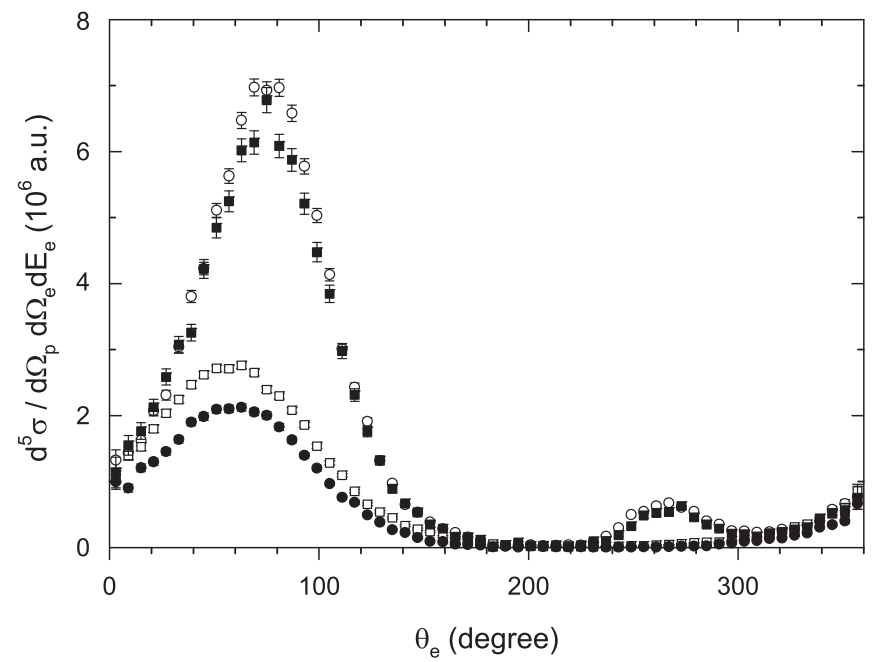

FIG. 4. FDCSs for the electron emission with $q=0.75$ a.u. and $E_{e}=6.5 \mathrm{eV}$ following the ionization of the helium atom by $1-\mathrm{MeV}$ protons in the scattering plane, as a function of the polar angle $\theta_{e}$. The data are results of the present CTMC calculations using different approximations. The traditional microcanonical CTMC is represented by open squares. The FDCSs obtained by the improved CTMC using hydrogenlike, RHF, and SPM wave functions are denoted by closed circles, closed squares, and open circles, respectively.

distribution: it does not predict the recoil peak. Similarly, no recoil peak was obtained by our improved CTMC based on Wigner distribution using the simplest, hydrogenlike trial wave function and potential. At the same time, the recoil peak appeared in the angular distributions obtained with the more realistic RHF and SPM functions, and applying GSZ potential.

The above results show the sensitivity of the FDCS calculations on the wave function and potential used for the determination of the initial position and momentum distribution of the electron. The failure of the hydrogenlike model can be traced back mainly to the underestimation of the spatial extension of the electron at larger distances (see Fig. 2). Furthermore, physically a good result is expected only if the average binding energy

$$
\langle u\rangle=\int_{u_{\min }}^{u_{\max }} d u u w(u)
$$

is close to the binding energy. This is not case for the hydrogenlike (single- $\zeta$ ) model: $\langle u\rangle=1.45$ a.u., while for the RHF and SPM models $\langle u\rangle$ has reasonable values, 0.86 and 0.93 a.u., respectively.

In the following discussions we present results obtained with the use of the SPM function. We refer to this model version as CTMC-SPM. In Fig. 5 for $1-\mathrm{MeV}$ proton impact we compare the result obtained by the latter model with the experimental data [2] and the predictions of quantummechanical models: the FBA and CDW-EIS-NN model of Voitkiv [7] and the 3C-SPM model of Chuluunbaatar et al. [9]. In CDW-EIS-NN the label "NN" refers to the inclusion of the internuclear interaction. In 3C-SPM the meaning of "SPM" is the same as in the present CTMC work: the authors of Ref. [9] applied the Silverman-Platas-Matsen function [34] for

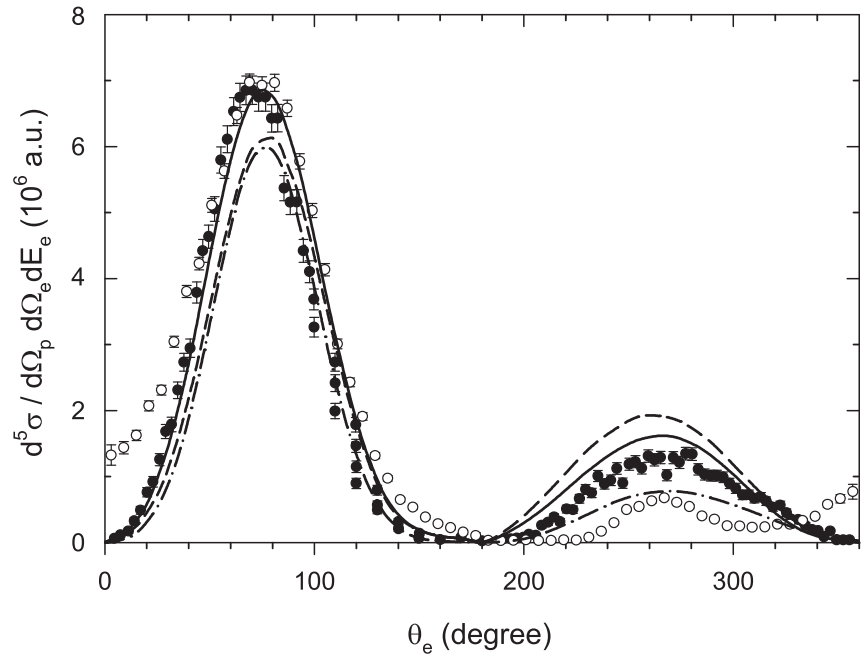

FIG. 5. Comparison of the FDCSs obtained by the present CTMC-SPM model (open circles) for $1-\mathrm{MeV}$ proton impact in the scattering plane with the experimental data [2] (closed circles) and the predictions of quantum-mechanical models: the FBA and CDWEIS-NN model of Voitkiv [7] (dashed and solid line, respectively) and the 3C-SPM model of Chuluunbaatar et al. [9] (dashed-dotted line). The experimental data are normalized to CDW-EIS-NN at the maximum of the binary peak.

the initial ground-state wave function of helium. We note that the latter authors have made calculations using also a strongly correlated function (CF) [35] which explicitly depends on the $r_{12}$ distance between electrons in helium. The result obtained with this 3C-CF model (not shown in Fig. 5) differs only slightly from that obtained with 3C-SPM.

Unlike in the present work and that of Ref. [9], Voitkiv [7] expressed the FDCS as differential with respect to the transverse momentum vector $\boldsymbol{Q}$ and electron momentum vector $\boldsymbol{k}$. We applied the following transformation between the two forms of FDCS:

$$
\frac{d^{5} \sigma}{d E_{e} d \Omega_{e} d \Omega_{p}}=p_{i}^{2} \cos \theta_{p} \sqrt{2 E_{e}} \frac{d^{5} \sigma}{d^{2} \boldsymbol{Q} d^{3} \boldsymbol{k}} .
$$

As mentioned above, no absolute FDCSs were measured in the experiment; therefore, we normalized the data to one of the theories, namely CDW-EIS-NN. The maximum of the binary peak was chosen as a normalization point. Focusing on the binary peak, above $40^{\circ} \mathrm{CTMC}-\mathrm{SPM}$ is in almost perfect agreement with CDW-EIS-NN and provides a reasonable description of the experimental data. The agreement is in absolute scale, no normalizing factor was applied to CTMCSPM. The FBA and 3C-SPM predict slightly smaller FDCS in the region of the binary peak. We note that the good agreement between CTMC-SPM and CDW-EIS-NN is partly explained by the fact that the core potential of helium used in Ref. [7] for the determination of the initial and final wave function agrees within $1 \%$ with the GSZ potential applied in the present work.

As mentioned in the Introduction, the shift of the observed binary peak towards smaller angles with respect to FBA indicates the presence of higher-order effects in the electron emission. To quantify the shift, we fitted a Gaussian to the 
TABLE I. Position of the binary peak and its shift with respect to FBA (in degrees) for $1 \mathrm{MeV}$ proton on helium collisions obtained from fittings of the measured and theoretically calculated FDCS data.

\begin{tabular}{lccc}
\hline \hline & Position & Error & Shift \\
\hline Experiment [2] & 71.01 & 0.34 & -6.6 \\
FBA [7] & 77.59 & 0.06 & \\
CDW-EIS-NN [7] & 75.89 & 0.08 & -1.7 \\
3C-SPM [9] & 76.79 & 0.05 & -0.9 \\
3C-CF [9] & 76.98 & 0.10 & -0.6 \\
CTMC-SPM (present) & 74.36 & 0.46 & -3.2 \\
\hline \hline
\end{tabular}

measured and theoretically calculated peaks in the angular range between $40^{\circ}$ and $110^{\circ}$.

The obtained peak positions, their errors and shifts are given in Table I. The fit was very good in all cases, as is seen from the errors obtained for the theoretical peaks. The larger errors at the experiment and CTMC are explained by the statistical scatterings of the data points. All the higher-order theories predict a shift towards smaller angles with respect to FBA; however, their predictions are considerably smaller than the observed shift. It is interesting that the best result (the largest shift) was achieved by the classical theory. We note here that there is a difference between the peak positions presented here and those given in Ref. [2] and mentioned in the Introduction. This can probably be explained by the different method used in Ref. [2] to determine the peak position, for example, by considering the entire peak region. However, we are mainly interested in the shift of the peak which is not so sensitive to the applied procedure. Indeed, the value $6^{\circ}$ given in Ref. [2] for the difference between the peak positions predicted by FBA and obtained experimentally agrees well with the corresponding value $6.6^{\circ}$ determined in the present work.

In contrast to the case of the binary peak, CTMC provides only a qualitative description of the recoil peak: the peak is smaller and narrower than that observed in the experiment and predicted by all the quantum-mechanical models. We note here that the recoil peak seems to be a challenge for the theory. From the very different results obtained by the models one may conclude a large sensitivity of the peak to the applied theoretical approach. At the same time, the disagreements between the quantum-mechanical models are only in respect to the amplitude of the peak; all the models predict a peak width in accordance with the experiment. The width obtained by CTMC is smaller by about a factor of two.

We searched for the reason of the failure of CTMC in reproducing the correct width of the recoil peak. It is plausible to assume that the peak, as a result of backscattering from the target nucleus, depends strongly on the electron-core interaction potential. Therefore, we made a series of trial calculations within the CTMC-SPM model applying different electron-core potentials. As a simple procedure, we changed the $\eta$ and $\xi$ parameters of the GSZ potential in a way that the screening of the target nuclear charge decreased by about $30 \%$ at typical electron distances $(\sim 1.2$ a.u. $)$. In another trial calculation we extended the GSZ potential by the polarization

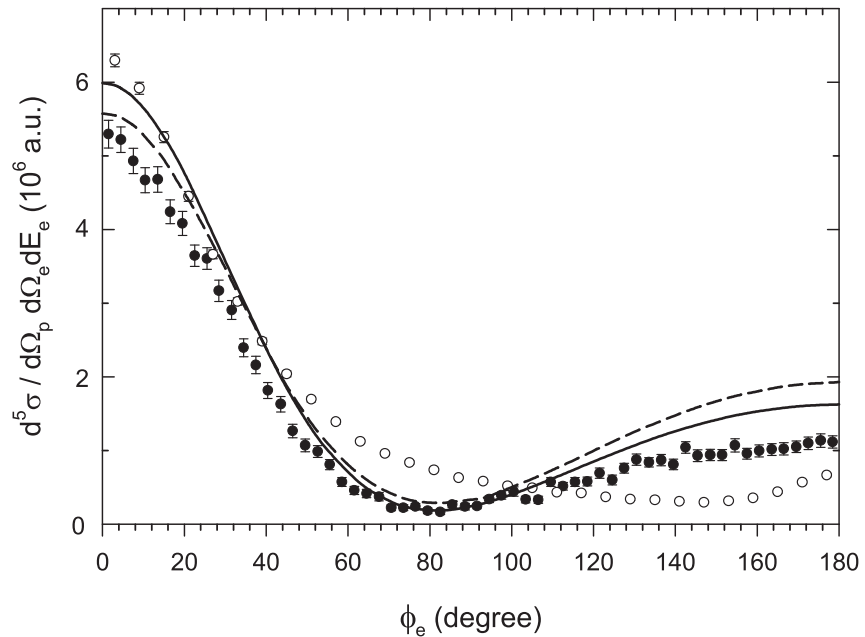

FIG. 6. FDCSs for $1-\mathrm{MeV}$ proton impact as a function of the azimuthal angle $\phi_{e}$ at a fixed value of the polar angle, $\theta_{e}=90^{\circ}$. The results of the present CTMC-SPM model (open circles) are compared with the experimental data [2] (closed circles) and the predictions of quantum-mechanical models: the FBA and the CDW-EIS-NN model of Voitkiv [7] (dashed and solid line, respectively).

potential proposed by Khan et al. [36]:

$$
V_{\mathrm{pol}}(r)=\left\{1-\exp \left[-\left(r / r_{c}\right)^{6}\right]\right\} \frac{\alpha}{2 r^{4}} .
$$

Here the parameters $r_{c}$ and $\alpha$ were taken from Refs. [36,37], respectively.

In a further calculation we extended the GSZ potential also by the so-called "Heisenberg core potential" suggested by Kirschbaum and Wilets [38] to prevent the classical manyelectron atom from autoionization:

$$
V_{\mathrm{H}}(r, p)=\frac{\kappa^{2}}{4 \beta r^{2}} \exp \left\{\beta\left[1-(r p / \kappa)^{4}\right]\right\} .
$$

Since $V_{\mathrm{H}}(r, p)$ depends on the momentum of the electron, for this potential we had to solve the canonical equations of motion for the three particles instead of the Newton's equations. We made a series of calculations using different values of the parameters $\kappa$ and $\beta$.

All the above modifications of the electron-core potential did not improve the description of the recoil peak; the peak remained narrow. This finding indicates that the formation of the recoil peak is probably dominated by quantum-mechanical effects that are consequences of the wave nature of the interacting particles (diffraction, interference). As a result, for example, the recoil peak shows much more sensitivity to the coherence property of the projectile beam than the binary peak (see a later discussion).

In Fig. 6 the present CTMC-SPM results are compared with the experimental data and quantum-mechanical calculations (FBA and CDW-EIS-NN [7]) as a function of the azimuthal angle $\phi_{e}$. In the CTMC calculations the azimuthal plane was fixed allowing electron emission in the polar angular range with $\theta_{e}=90^{\circ} \pm 5^{\circ}$. The experimental data in the figure are normalized in the same way as in Fig. 5. Again, at small angles CTMC agrees well with CDW-EIS-NN. Above $40^{\circ}$, however, it shows large deviations from both the experiment and the quantum-mechanical theories. Particularly, CTMC 


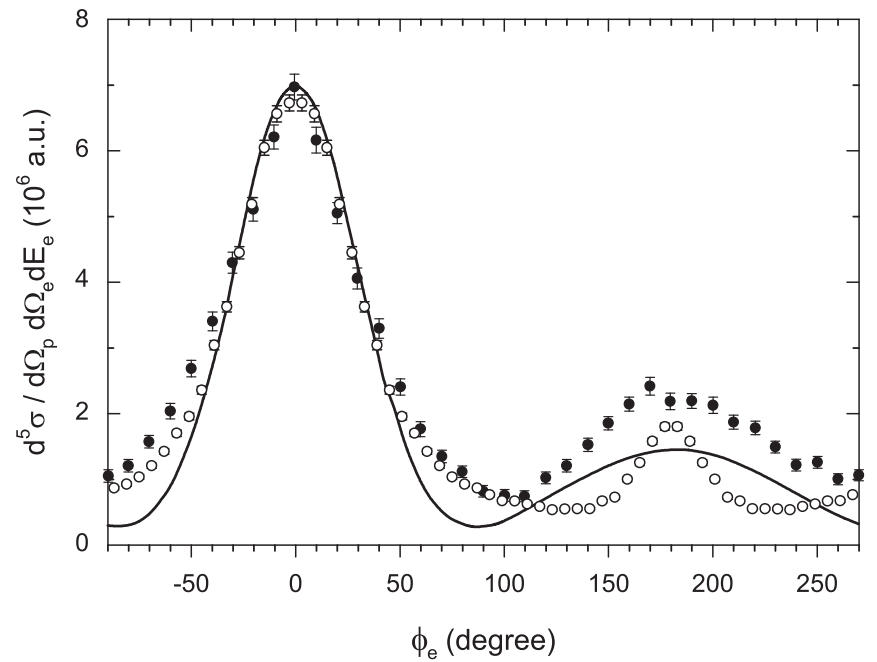

FIG. 7. Comparison of the FDCSs obtained by the present CTMC-SPM model (open circles) with the experimental data [6] (closed circles) and the results of CDW-EIS calculations [6] (solid line) for $3-\mathrm{MeV}$ proton impact in the azimuthal plane. The experimental data are normalized to CDW-EIS at the maximum of the binary peak.

gives a poor account of the minimum of the FDCS observed in the experiment at $\phi_{e} \approx 80^{\circ}$ : the minimum is shifted to a much larger angle $\left(\phi_{e} \approx 150^{\circ}\right)$, and it is less pronounced.

In a further analysis we checked the performance of CTMC at higher proton energy. In Fig. 7 we compare the angular distribution of FDCSs obtained by CTMC-SPM for 3-MeV collisions in the azimuthal plane with the measured data of Wang et al. [6] and the predictions of a CDW-EIS model [6] which include the NN interaction within the eikonal approximation [39]. The momentum transfer and the electron energy are the same as in the $1-\mathrm{MeV}$ experiment, $q=0.75 \mathrm{a}$.u. and $E_{e}=6.5 \mathrm{eV}$, respectively. Here again we normalized the experimental data to CDW-EIS in the same way as in Fig. 5. From the excellent agreement seen between CTMC-SPM and CDW-EIS at the maximum of the binary peak here and also at $1 \mathrm{MeV}$ impact we may conclude that the classical theory follows well the energy dependence of the quantummechanical description. The observed shape of the binary peak is well reproduced by CTMC. Our calculations resulted in again a narrow recoil peak, but now it is more pronounced than that obtained at $1-\mathrm{MeV}$ impact energy.

As a particular interesting check of the present CTMC model, we considered the collisions of $100-\mathrm{MeV} / \mathrm{u} \mathrm{C}^{6+}$ ions with helium. The experiment made on this system [3] resulted in FDCS data in a serious discrepancy with the quantummechanical theories. While in the scattering plane the angular distribution of the measured data agreed well with the theoretical prediction; in the plane perpendicular to the momentum transfer a strong disagreement was found: the measurement did not show the anticipated node structure mentioned in the Introduction. This so-called " $\mathrm{C}^{6+}$ puzzle" initiated a large number of theoretical investigations [6,14,39-52].

In Fig. 8 we compare the FDCSs obtained by the present CTMC model for $100-\mathrm{MeV} / \mathrm{u} \mathrm{C}^{6+}$ projectile ions with the experimental data and the results of the following theoretical

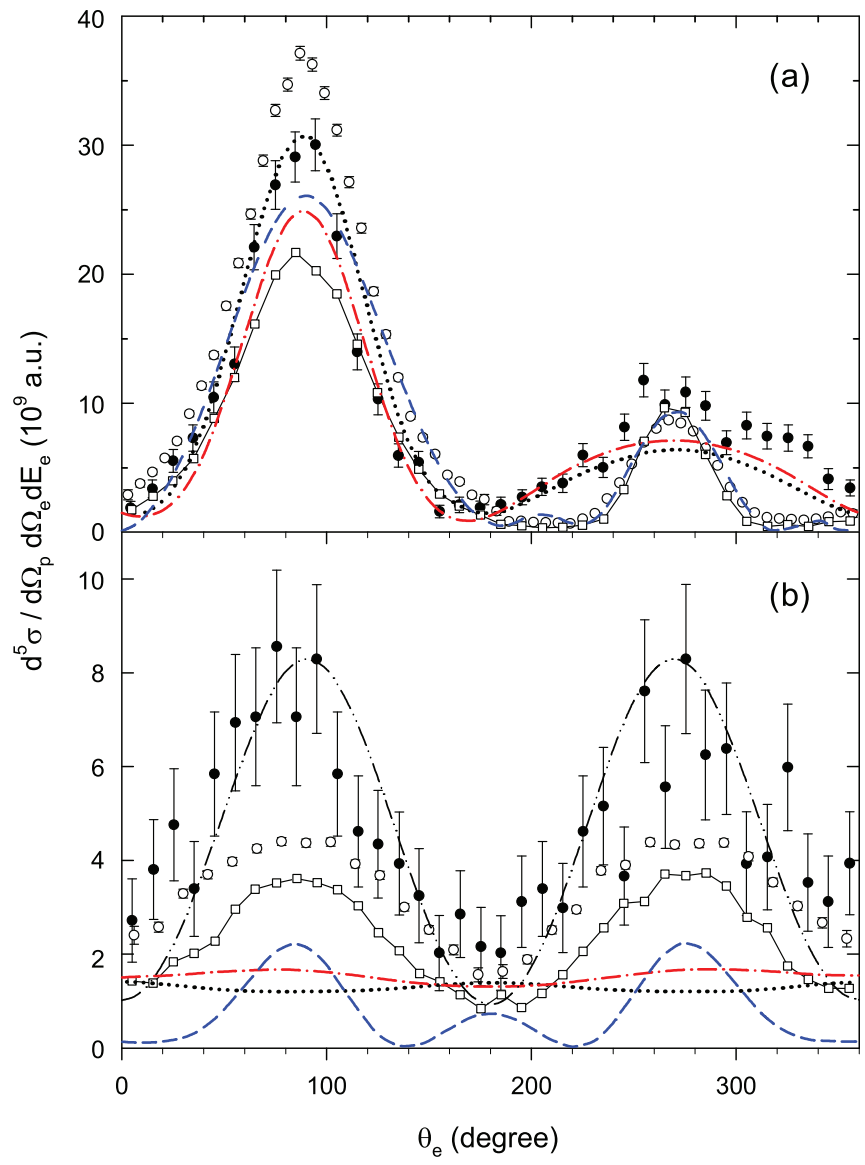

FIG. 8. Experimental and theoretical FDCSs for $100-\mathrm{MeV} / \mathrm{u} \mathrm{C}^{6+}$ ion impact in the scattering plane (a) and in the perpendicular plane (b). The notations: closed circles, experimental data [3]; open circles, present CTMC-SPM; open squares connected with line, the CTMC results of Olson and Fiol [14]; dotted line, CDW [3]; dashed-line (blue), incoherent SCA [51]; dashed-dotted line (red), coherent SCA [51]; dashed-dotted-dotted line, wave-packet FBA [52] (see text).

models: a three-body Wigner-type CTMC model [14], a version of the CDW description [3], and a semiclassical (SCA) model proposed by Járai-Szabó and Nagy [46,51]. Again, collisions with $q=0.75$ a.u. and $E_{e}=6.5 \mathrm{eV}$ are considered and the experimental data are normalized to CDW at the maximum of the binary peak.

The SCA model $[46,51]$ is of special importance from the point of view of the present work; therefore, we briefly summarize it. The model was developed for the investigation of the effect of the so-called projectile beam coherence on the single ionization of atoms by ion impact. In the past few years the beam coherence has been the subject of several experimental and theoretical investigations (for a review, see Ref. [53]). The increased activity in this field was triggered by the pioneering experiment of Egodapitiya et al. [54], in which it was shown that by suitable manipulation of the ion beam its coherence properties can be controlled.

The model is an impact-parameter description of the ionization assuming a straight-line projectile path. The transition amplitude from the initial bound state $|i\rangle$ to a final continuum state $|f\rangle$ is determined using the time-dependent perturbation 
theory. The first-order approximation results in

$$
a^{(1)}(\boldsymbol{B})=-\frac{i}{v} \int_{-\infty}^{+\infty} d z \exp \left(i \frac{E_{f}-E_{i}}{v} z\right)\langle f|V(\boldsymbol{B})| i\rangle,
$$

where $E_{i}$ and $E_{f}$ are the energies of the initial and final state, $V(\boldsymbol{B})$ is the projectile-electron interaction potential, $v$ is the projectile velocity, and $\boldsymbol{B}$ is the impact parameter vector. Angular momentum dependent amplitudes, $a_{l_{f}, m_{f}}^{(1)}(\boldsymbol{B})$, are obtained by expanding $|f\rangle$ into partial waves.

The FDCS is calculated in two ways, incoherently and coherently. The incoherent expression has the form

$$
\frac{d^{5} \sigma_{\mathrm{inc}}}{d E_{e} d \Omega_{e} d \boldsymbol{q}_{\perp}}=B\left|\frac{d B}{d \theta_{p}}\right|\left|\sum_{l_{f}, m_{f}} a_{l_{f}, m_{f}}^{(1)}(\boldsymbol{B})\right|^{2} .
$$

In the evaluation of Eq. (12) the relation between $\boldsymbol{B}$ and $\boldsymbol{q}_{\perp}$ has to be established. The authors of Ref. [51] made it in two steps. First, they determined the relationship between $\theta_{p}$ and $q_{\perp}$ on the basis of the transverse momentum balance of the collision. Here they assumed $\boldsymbol{q}_{\perp} \approx \boldsymbol{q}$ which is justified at the considered high-velocity collision. Second, they determined $\theta_{p}$ as a function of $B$ by solving classically the problem of the elastic scattering of the projectile on the screened Coulomb potential of the target nucleus. Since a single impact parameter belongs to a given transverse momentum, no coherence exists between the different impact parameters.

For derivation of the coherent expression of FDCS the authors of Ref. [51] considered the relationship between the $\boldsymbol{q}_{\perp}$ - and $\boldsymbol{B}$-dependent transition amplitudes known from the quantum scattering theory [55]:

$$
R\left(\boldsymbol{q}_{\perp}\right)=\frac{1}{2 \pi} \sum_{l_{f}, m_{f}} \int d \boldsymbol{B} e^{i \boldsymbol{B} \boldsymbol{q}_{\perp}} B^{2 i \frac{z_{p} Z_{t}}{v}} a_{l_{f}, m_{f}}^{(1)}(\boldsymbol{B}) .
$$

Here the factor $B^{2 i \frac{z_{p} Z_{t}}{v}}$ gives account of the effect of the nucleus-nucleus interaction. The FDCS is then obtained as

$$
\frac{d^{5} \sigma_{\mathrm{coh}}}{d E_{e} d \Omega_{e} d \boldsymbol{q}_{\perp}}=p_{i}\left|R\left(\boldsymbol{q}_{\perp}\right)\right|^{2} .
$$

Equations (13) and (14) provide a solution that is equivalent to the full quantum mechanical solution based on planewave representation of the projectile. Physically, according to Eq. (13) the contributions of the different impact parameters are added coherently.

Turning back to Fig. 8, in the scattering plane [see panel (a)] the quantum-mechanical theories-CDW and the coherent SCA-are in qualitative agreement with the experiment. Both CTMC calculations reproduce well the observed shape of the binary peak. It is a remarkable achievement of the present CTMC model that even for such a high-velocity and highly charged projectile it predicts only slightly different FDCS values in the region of the binary peak than CDW. In this region the CTMC results of Olson and Fiol [14] are smaller than the present results by almost a factor of two. The difference between the two models can be explained by the fact that the procedure of Olson and Fiol was based on a discrete sum of the microcanonical distribution expressed by Eq. (6). Furthermore, the latter authors used a less realistic initial-state helium wave function than the SPM considered in the present work.

For the recoil peak both CTMC calculations yielded a narrow peak in strong disagreement with the experiment and the quantum-mechanical descriptions. The incoherent SCA predicts also a narrow peak, in an excellent agreement with the CTMC models. A striking feature of Fig. 8(a) is the large difference between the results of the coherent and incoherent SCA for the recoil peak. The finding that the observed broad peak is reproduced by the coherent sum of the contributions of the different impact parameters [see Eq. (13)] confirms our guess that the formation of the recoil peak is strongly influenced by quantum-mechanical effects.

In Fig. 8(b) we compare the measured and calculated FDCSs as a function of the polar angle of the electron in the $y z$ plane. Since for the considered high-velocity collision the momentum transfer is almost perpendicular to the initial momentum of the projectile, this plane is also called the "perpendicular" plane. According to the figure, the quantum-mechanical descriptions are in a complete disagreement with the experiment; they failed to reproduce the peaks at $90^{\circ}$ and $270^{\circ}$. At the same time, the peaks appear in the angular distribution predicted by both CTMC models and the incoherent SCA. Again, we call attention to the large difference between the results of the coherent and incoherent SCA.

A possible explanation of the observed peaks in the perpendicular plane based on the analysis of the classical particle trajectories is given in Ref. [14].

The appearance of the peaks in the CTMC and the incoherent SCA calculations indicates the presence of projectile coherence effects in the collision, namely the loss of coherence. To take into account this effect, Navarrete et al. [52] performed FBA calculations in which the motion of the projectile was represented by a wave packet instead of plane wave [56,57]. For comparison, they made the calculations also with plane wave, i.e., for the case of complete projectile coherence. The latter calculations resulted in similar angular distributions of FDCS as those predicted by CDW and the coherent SCA. In the absence of reliable knowledge of the coherence properties of the projectile beam applied in the experiment, Navarrete et al. [52] considered the momentum spread of the wave packet as a free parameter and fitted the FBA results to the experimental data in both the collision and perpendicular plane. As it is seen in Fig. 8(b), a very good fit was obtained in the perpendicular plane, i.e., the measured FDCS could be well reproduced assuming a partial coherence for the projectile beam. In the scattering plane at the same value of the fitting parameter the wave-packet calculations resulted in only slightly different FDCSs [not shown in Fig. 8(a)] than those obtained by the traditional, plane-wave FBA.

The wave-packet description assumes a kind of localization of the projectile. This suggests the picture that with increasing incoherence the projectiles tend to behave classically in the ionization process. The finding in the perpendicular plane that by considering the loss of coherence in FBA a qualitative agreement was achieved with both CTMC models and the experiment seems to support the above picture. However, it is important to emphasize that in general an incoherent particle beam cannot be regarded as an ensemble of classical particles. A good example of this is the small change of the recoil 

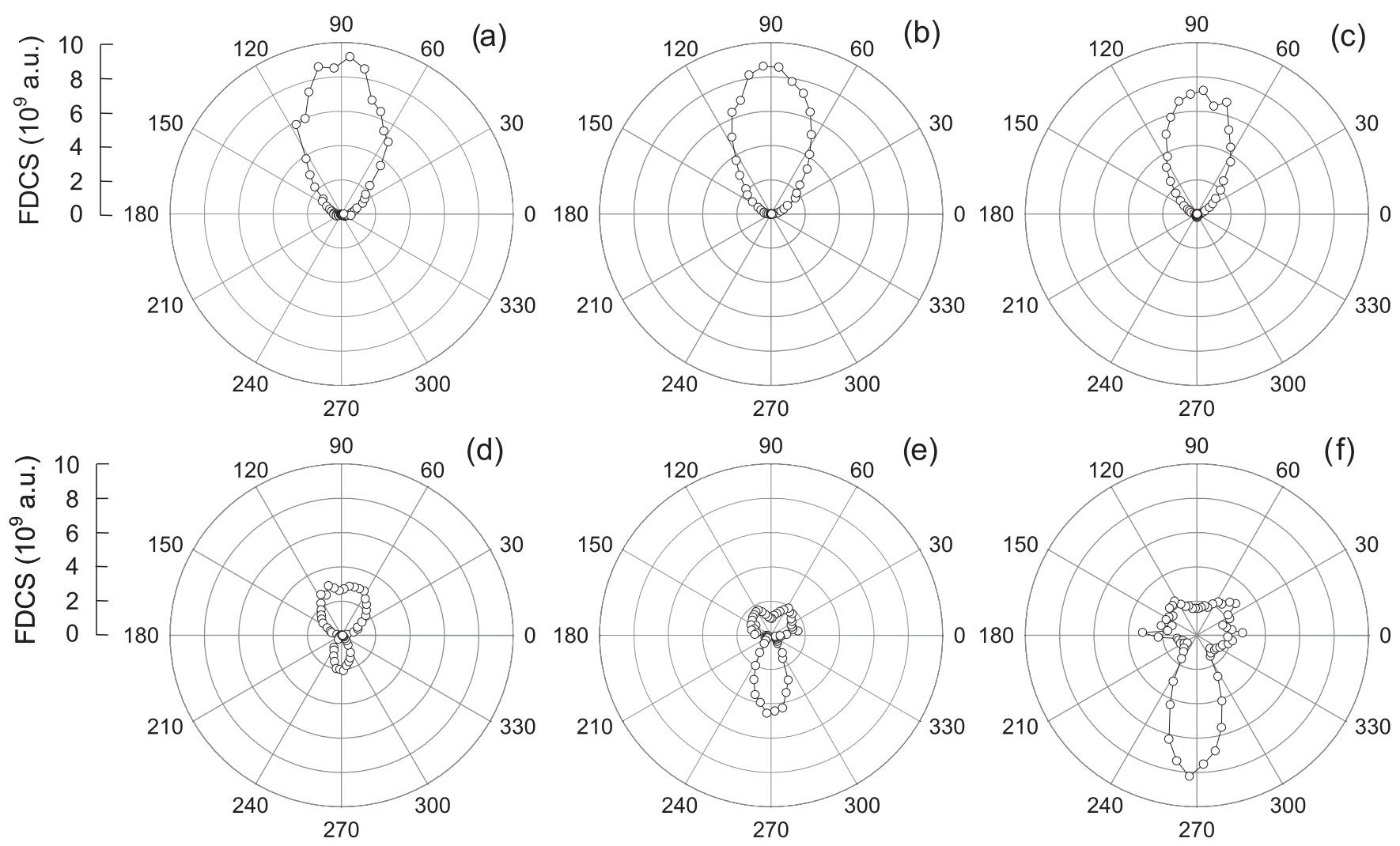

FIG. 9. Polar diagrams of the angular distribution of the electron for $100-\mathrm{MeV} / \mathrm{u} \mathrm{C}^{6+}$ ion impact in scattering planes chosen at angles $\phi_{q}=0^{\circ}(\mathrm{a}), 45^{\circ}(\mathrm{b}), 90^{\circ}$ (c), $135^{\circ}$ (d), $160^{\circ}$ (e), and $180^{\circ}$ (f) relative to the initial collision plane.

peak in the scattering plane obtained in the wave-packet FBA calculations (see Fig. 1 in Ref. [52])—for classical projectiles the same narrow peak would be expected as that obtained by the CTMC models and the incoherent SCA.

The present CTMC work led to the recognition of the following classical property of the FDCS. In the quantummechanical treatment of the collision the scattering of the projectile is unambiguously characterized by the scattering plane defined by the $\boldsymbol{p}_{i}$ and $\boldsymbol{p}_{f}$ vectors. Classically, besides this final scattering plane there exists also the initial collision plane defined by the $\boldsymbol{p}_{i}$ and $\boldsymbol{B}$ vectors. This means that a more differential FDCS can be defined which depends also on the angle of the initial collision plane determined by the azimuthal angle of $\boldsymbol{B}$ :

$$
\frac{d^{5} \sigma}{d E_{e} d \Omega_{e} d \Omega_{p}} \rightarrow \frac{d^{6} \sigma}{d E_{e} d \Omega_{e} d \Omega_{p} d \phi_{B}} .
$$

We calculated the above extended FDCSs in the case of $100 \mathrm{MeV} / \mathrm{u} \mathrm{C}^{6+}+\mathrm{He}$ collision. In Fig. 9 we presented the results in a way that we fixed the angle of the initial collision plane at $\phi_{B}=0$ and chose five scattering planes at azimuthal angles $\phi_{q}=0^{\circ}, 45^{\circ}, 90^{\circ}, 135^{\circ}, 160^{\circ}$, and $180^{\circ}$. Deviations $\Delta \phi_{q}= \pm 10^{\circ}$ were allowed. We considered the electron emission in these planes and plotted the FDCS as a function of $\theta_{e}$.

According to Fig. 9, up to $\phi_{q}=135^{\circ}$ the binary peak dominates the angular distribution. At this angle the recoil peak also appears, and at $\phi_{q}=180^{\circ}$ it becomes the dominant component.

The above analysis has the following importance in the understanding of the ionization mechanism. It gives information about to what extent the three-body collision can be regarded as a two-body scattering of the projectile on the target in the sense that the pure two-body scattering in a central potential classically is a plane motion, i.e., the initial plane coincides with the scattering plane. We note here that this assumption was made in Ref. [51] in the evaluation of Eq. (12). There are two extreme cases. (i) The projectile interacts dominantly with the electron; the target core is only a spectator. This case can be best visualized by the simplified picture of scattering of the projectile on a quasifree electron in which the role of the scattering center is taken over by the electron. For such collisions $\boldsymbol{B}$ ceases to be a relevant quantity; the correlation between the scattering plane and the initial collision plane is small. Since the scattering center (the electron) is moving, the scattering is diffuse; the projectile may scatter out from the initial plane in any directions. This is the case of the binary peak: according to Fig. 9, the angular distribution of the binary peak is practically constant between $\phi_{q}=0^{\circ}$ and $90^{\circ}$, i.e., the probability of the outscattering into the different scattering planes is almost the same in this broad range. (ii) When the projectile transfers momentum dominantly to the target core (directly by the N-N, or indirectly by the N-e-N interaction), the collision has two-body scattering character in respect to the projectile and the whole atom. In this case the probability of the 
outscattering of the projectile from the initial collision plane is small; the electron emission is strongly peaked at $\phi_{q}=0^{\circ}$ or $\phi_{q}=180^{\circ}$, depending on the sign of the interaction (attractive or repulsive). Such a behavior is expected for the recoil peak because of the large momentum transferred to the target core. Indeed, the recoil peak emerges suddenly as a function of $\phi_{\boldsymbol{q}}$ and has a maximum at $180^{\circ}$, as it is seen in Fig. 9.

On the basis of the above analysis we point to a deficiency of the expression (12) proposed by Járai-Szabó and Nagy [51] for the calculation of FDCS for an incoherent projectile beam. The authors considered the case (ii) when they determined the $B\left(\theta_{p}\right)$ function solving classically the problem of the elastic scattering of the projectile on the screened Coulomb potential of the target nucleus. The assumption of isotropy for the relationship between $\boldsymbol{q}_{\perp}$ and $\boldsymbol{B}$ in Eq. (12) is likely a rough approximation. Obviously, the use of the same $B\left(\theta_{p}\right)$ function for case (i), i.e., for collisions dominated by the electron-projectile interaction, is highly questionable.

Since the impact parameter is not an observable quantity, the extended FDCS differential with respect $\phi_{\boldsymbol{B}}$ cannot be measured. The concept of the initial scattering plane has a meaning only in the classical description of the collision. Nevertheless, in quantum mechanics the representation of the projectile by a wave packet allows a kind of initial preparation of the projectile beam. Considering the relationship between the concept of the wave packet and the coherence property of the projectile, some information about the dynamics discussed in the context of Fig. 9 can be obtained in an experiment carried out with a projectile beam of anisotropic coherence, namely with a beam characterized by different transverse coherence lengths along two mutually perpendicular directions. Let us assume a beam, for example, with a large coherence length (compared to the size of the He atom) along the $x$ axis in the laboratory coordinate system, and a very small coherence length along the $y$ axis. This defines a distinguished physical direction in the space. By taking the $x$ axis for this direction, the extension of FDCS means that it can be measured also as a function of $\phi_{q}$, the angle of the scattering plane.

We note that in the experiment of Gassert et al. [2] the proton beam had the same type of anisotropic coherence as that discussed above. The authors utilized this property of the beam in an attempt to show the effect of the coherence on the FDCS. To this end they determined the angular distribution of the electron in two scattering planes that were taken in the direction of the large and small transverse coherence length. Agreement was found between the two angular distributions from which the authors concluded that the projectile coherence did not influence the FDCS. However, according to Schulz [53] the result of the experiment was not conclusive, because the coherence length in both directions was larger than the size of the helium atom.

\section{CONCLUSIONS}

We investigated the performance of the CTMC method in the calculation of FDCS for the ion-induced single ionization of helium. We showed that a reasonable agreement with the experimental data can only be achieved by approaches that use an approximate Wigner function for the determination of the initial position and momentum distribution of the atomic electron. We worked out a Wigner-type CTMC model in which the quantum-mechanical position distribution is approximated by a weighted integral of the microcanonical distribution over a range of the binding energy of the electron. This is a generalization of a previous model based on a discrete sum of weighted microcanonical distributions.

We made a series of calculations within the proposed model using different helium ground-state wave functions and electron-target core potentials. We found a strong dependence of the FDCS on the wave function and potential. The best performance was obtained with the SPM wave function [34] and the GSZ potential [27].

We compared the predictions of the CTMC-SPM model with the experimental data and other theoretical results for 1and $3-\mathrm{MeV}$ proton and $100-\mathrm{MeV} / \mathrm{u} \mathrm{C}^{6+}$ ion impact. In the scattering plane both the classical and quantum-mechanical theory reproduced well the shape of the binary peak. In the region of the binary peak a good agreement was found on absolute scale between the present CTMC model and available CDW calculations for all the investigated collisions. This is a remarkable result, considering that the two theoretical approaches are completely different. For $1 \mathrm{MeV}$-proton impact we investigated in detail the shift of the binary peak with respect to FBA. It was found that the shifts predicted by the higher-order theories were considerably smaller than the observed one. In this respect the best result (the largest shift) was achieved by the present CTMC calculations.

Both the present and a previous CTMC model failed to reproduce the shape of the recoil peak observed in the experiments, the classical theory predicts a much narrower peak. From this discrepancy we concluded that the formation of the recoil peak is probably strongly influenced by quantummechanical effects. We drew the same conclusion from the large difference between the shapes of the peak calculated with the coherent and incoherent SCA model in the case of the $100-\mathrm{MeV} / \mathrm{u} \mathrm{C}^{6+}$ ion impact. For the latter collision the present CTMC calculations confirmed the existence of the "double-peak" structure of the angular distribution of the electron in the perpendicular plane, in accordance with the observation and previous CTMC results. This finding together with wave-packet calculations suggests that the " $\mathrm{C}^{6+}$ puzzle" may be solved by considering the loss of the projectile coherence.

Finally, we analyzed the three-body dynamics of the ionization by introducing the concept of the initial collision plane. Although this concept proved to be useful in the deeper understanding of the dynamics, we stressed that it has a meaning only in the classical description of the collision. At the same time, our analysis called attention to a possible extension of FDCS by measuring it relative to a distinguished physical direction. We considered the anisotropic coherence of the projectile ion beam, which may define a direction in the space.

\section{ACKNOWLEDGMENTS}

This work was supported by the National Scientific Research Foundation (OTKA, Grant No. K109440) and the National Information Infrastructure Program (NIIF). 
TABLE II. Parameters of the Roothaan-Hartree-Fock groundstate wave function of helium [33].

\begin{tabular}{lcc}
\hline \hline$i$ & $C_{i}$ & $\zeta_{i}$ \\
\hline 1 & 0.76838 & 1.41714 \\
2 & 0.22346 & 2.37682 \\
3 & 0.04082 & 4.39628 \\
4 & -0.00994 & 6.52699 \\
5 & 0.00230 & 7.94252 \\
\hline
\end{tabular}

APPENDIX: POSITION AND MOMENTUM
DISTRIBUTIONS DERIVED FROM THE TRIAL GROUND-STATE HELIUM WAVE FUNCTIONS AND THE OPTIMIZED WEIGHT FUNCTIONS IN THE INTEGRAL IN EQ. (5)

\section{Hydrogenlike (single- $\zeta$ ) wave function}

The position and momentum distributions:

$$
\begin{gathered}
\rho_{\mathrm{H}}(r)=4 Z_{\mathrm{eff}}^{3} r^{2} \exp \left(-2 Z_{\mathrm{eff}} r\right), \\
\rho_{\mathrm{H}}(p)=\frac{32}{\pi} \frac{Z_{\mathrm{eff}}^{5}}{\left(Z_{\mathrm{eff}}^{2}+p^{2}\right)^{4}} p^{2},
\end{gathered}
$$

with $Z_{\text {eff }}=2-5 / 16$. The fit of the integral (5) to $\rho_{\mathrm{H}}(r)$ was made with the Coulomb potential $V(r)=-Z_{\text {eff }} / r$ in Eq. (4). The optimized weight function:

$$
\begin{aligned}
& w(u)=15.56 u^{3}\left[1-\exp \left(-5 u^{2}\right)\right]^{4} \exp (-3 u), \\
& u_{\min }=0.32 \text { a.u., } \quad u_{\max }=4.52 \text { a.u. }
\end{aligned}
$$

\section{Roothaan-Hartree-Fock (RHF) [33] wave function}

The position distribution:

$$
\rho_{\mathrm{RHF}}(r)=\frac{1}{2}\left[\sum_{i=1}^{5} C_{i}\left(2 \zeta_{i}\right)^{3 / 2} \exp \left(-\zeta_{i} r\right)\right]^{2} r^{2}
$$

The values of $C_{i}$ and $\zeta_{i}$ are listed in Table II.

The fit of the integral (5) to $\rho_{\mathrm{RHF}}(r)$ was made with the GSZ potential [27] in Eq. (4). The optimized weight function:

$$
\begin{aligned}
& w(u)=1794 u^{2}\left[1-\exp \left(-11.8 u^{2}\right)\right]^{4} \exp (-8 \sqrt{u}), \\
& u_{\min }=0.175 \text { a.u., } \quad u_{\max }=4.52 \text { a.u. }
\end{aligned}
$$

The derivation of the momentum distribution was not straightforward. The RHF wave function proposed by Clementi and Roetti is built from Slater-type orbitals (STOs). The momentum representations of STOs are not so well known. Komarov and Remkin [58] derived the following expressions for the $1 s$ and $2 p$ orbitals:

$$
\begin{aligned}
\chi_{1 s}(p) & =\frac{2^{3 / 2}}{\pi} \frac{\zeta^{5 / 2}}{\left(\zeta^{2}+p^{2}\right)^{2}}, \\
\chi_{2 p}(p) & =\frac{2^{7 / 2} \zeta^{7 / 2}}{3^{1 / 2} \pi} \frac{p}{\left(\zeta^{2}+p^{2}\right)^{3}} .
\end{aligned}
$$

The normalizations of the above functions, however, are erroneous. The correct formulas:

$$
\begin{aligned}
\chi_{1 s}(p) & =\frac{2^{5 / 2}}{\sqrt{\pi}} \frac{\zeta^{5 / 2}}{\left(\zeta^{2}+p^{2}\right)^{2}}, \\
\chi_{2 p}(p) & =\frac{2^{9 / 2}}{\sqrt{3 \pi}} \frac{\zeta^{7 / 2} p}{\left(\zeta^{2}+p^{2}\right)^{3}} .
\end{aligned}
$$

With the above corrected $\chi_{1 s}(p)$ function the RHF momentum distribution is expressed as

$$
\rho_{\mathrm{RHF}}(p)=\frac{32}{\pi}\left[\sum_{i=1}^{5} C_{i} \frac{\zeta^{5 / 2}}{\left(\zeta^{2}+p^{2}\right)^{2}}\right]^{2} p^{2} .
$$

Here the parameters $C_{i}$ and $\zeta_{i}$ are the same as in Eq. (A3). The values of $C_{i}$ and $\zeta_{i}$ are listed in Table II.

\section{Silverman-Platas-Matsen (SPM) [34] wave function}

The position distribution:

$$
\begin{aligned}
\rho_{\mathrm{SPM}}(r)= & \frac{1}{\left(1+\lambda^{2}\right)}\left\{N ^ { 2 } \left[R_{10}^{2}(r)+R_{10}^{\prime 2}(r)\right.\right. \\
& \left.\left.+2 R_{10}(r) R_{10}^{\prime}(r) S\right]+\lambda^{2} R_{21}^{2}(r)\right\} r^{2},
\end{aligned}
$$

with

$$
\begin{aligned}
R_{10}(r) & =2 a^{3 / 2} \exp (-a r), \\
R_{10}^{\prime}(r) & =2 b^{3 / 2} \exp (-b r), \\
R_{21}(r) & =(2 / \sqrt{3}) g^{5 / 2} r \exp (-g r), \\
S & =\int_{0}^{\infty} R_{10}(r) R_{10}^{\prime} r^{2} d r=8(a b)^{3 / 2} /(a+b)^{3}, \\
N & =\left(2+S^{2}\right)^{-1 / 2} .
\end{aligned}
$$

Here $\lambda=-0.0617557, a=2.17621, b=1.20152$, and $g=$ 2.47547. The fit of the integral (5) to $\rho_{\mathrm{SPM}}(r)$ was made with the GSZ potential [27] in Eq. (4). The optimized weight function:

$$
\begin{aligned}
& w(u)=7305 u^{3}\left[1-\exp \left(-19 u^{2}\right)\right]^{4} \exp (-9.4 \sqrt{u}), \\
& u_{\min }=0.16 \text { a.u., } \quad u_{\max }=4.52 \text { a.u. }
\end{aligned}
$$

The momentum distribution:

$$
\begin{aligned}
\rho_{\mathrm{SPM}}(p)= & \frac{1}{\left(1+\lambda^{2}\right)}\left\{N ^ { 2 } \left[\tilde{R}_{10}^{2}(p)+\tilde{R}_{10}^{\prime 2}(p)\right.\right. \\
& \left.\left.+2 \tilde{R}_{10}(p) \tilde{R}_{10}^{\prime}(p) S\right]+\lambda^{2} \tilde{R}_{21}^{2}(p)\right\} p^{2},
\end{aligned}
$$

with

$$
\begin{aligned}
\tilde{R}_{10}(p) & =\frac{2^{5 / 2}}{\sqrt{\pi}} \frac{a^{5 / 2}}{\left(a^{2}+p^{2}\right)^{2}}, \\
\tilde{R}_{10}^{\prime}(p) & =\frac{2^{5 / 2}}{\sqrt{\pi}} \frac{b^{5 / 2}}{\left(b^{2}+p^{2}\right)^{2}}, \\
\tilde{R}_{21}(p) & =\frac{2^{9 / 2}}{\sqrt{3 \pi}} \frac{g^{7 / 2} p}{\left(g^{2}+p^{2}\right)^{3}}, \\
S & =\int_{0}^{\infty} \tilde{R}_{10}(r) \tilde{R}_{10}^{\prime} p^{2} d p=8(a b)^{3 / 2} /(a+b)^{3}, \\
N & =\left(2+S^{2}\right)^{-1 / 2} .
\end{aligned}
$$

Here we used Eqs. (A4) and (A5). The parameters $\lambda, a, b$, and $g$ are the same as for $\rho_{\mathrm{SPM}}(r)$. 
[1] R. Dörner, V. Mergel, O. Jagutzki, L. Spielberger, J. Ullrich, R. Moshammer, and H. Schmidt-Böcking, Phys. Rep. 330, 95 (2000).

[2] H. Gassert, O. Chuluunbaatar, M. Waitz, F. Trinter, H.-K. Kim, T. Bauer, A. Laucke, Ch. Müller, J. Voigtsberger, M. Weller, J. Rist, M. Pitzer, S. Zeller, T. Jahnke, L. Ph. H. Schmidt, J. B. Williams, S. A. Zaytsev, A. A. Bulychev, K. A. Kouzakov, H. Schmidt-Böcking, R. Dörner, Yu. V. Popov, and M. S. Schöffler, Phys. Rev. Lett. 116, 073201 (2016).

[3] M. Schulz, R. Moshammer, D. Fischer, H. Kollmus, D. H. Madison, S. Jones, and J. Ullrich, Nature (London) 422, 48 (2003).

[4] D. Fischer, M. Schulz, R. Moshammer, and J. Ullrich, J. Phys. B: At., Mol., Opt. Phys. 37, 1103 (2004).

[5] M. Schulz, R. Moshammer, A. Voitkiv, B. Najjari, and J. Ullrich, Nucl. Instrum. Methods, Phys. Res. B 235, 296 (2005).

[6] X. Wang, K. Schneider, A. LaForge, A. Kelkar, M. Grieser, R. Moshammer, J. Ullrich, M. Schulz, and D. Fischer, J. Phys. B: At., Mol., Opt. Phys. 45, 211001 (2012).

[7] A. B. Voitkiv, Phys. Rev. A 95, 032708 (2017).

[8] F. Martin and A. Salin, Phys. Rev. A 55, 2004 (1997).

[9] O. Chuluunbaatar, S. A. Zaytsev, K. A. Kouzakov, A. Galstyan, V.L. Shablov, and Yu. V. Popov, Phys. Rev. A 96, 042716 (2017).

[10] M. Brauner, J. S. Briggs, and H. Klar, J. Phys. B: At., Mol., Opt. Phys. 22, 2265 (1989).

[11] M. Schulz, R. Moshammer, D. H. Madison, R. E. Olson, P. Marchalant, C. T. Whelan, H. R. J. Walters, S. Jones, M. Foster, H. Kollmus, A. Cassimi, and J. Ullrich, J. Phys. B: At., Mol., Opt. Phys. 34, L305 (2001).

[12] J. Fiol and R. E. Olson, J. Phys. B: At., Mol., Opt. Phys. 35, 1759 (2002).

[13] J. Fiol and R. E. Olson, Nucl. Instrum. Methods, Phys. Res. B 205, 474 (2003).

[14] R. E. Olson and J. Fiol, J. Phys. B: At., Mol., Opt. Phys. 36, L365 (2003).

[15] J. Fiol and R. E. Olson, J. Phys. B: At., Mol., Opt. Phys. 37, 3947 (2004).

[16] R. E. Olson and J. Fiol, Phys. Rev. Lett. 95, 263203 (2005).

[17] S. Otranto, R. E. Olson, and J. Fiol, J. Phys. B: At., Mol., Opt. Phys. 39, L175 (2006).

[18] L. Sarkadi and L. Gulyás, Phys. Rev. A 90, 022702 (2014).

[19] R. Abrines and I. C. Percival, Proc. Phys. Soc. London 88, 861 (1966).

[20] T. Geyer and J. M. Rost, J. Phys. B: At., Mol., Opt. Phys. 34, L47 (2001).

[21] T. Geyer and J. M. Rost, J. Phys. B: At., Mol., Opt. Phys. 35, 1479 (2002).

[22] D. Eichenauer, N. Grün, and W. Scheid, J. Phys. B: At. Mol. Phys. 14, 3929 (1981).

[23] D. J. W. Hardie and R. E. Olson, J. Phys. B: At. Mol. Phys. 16, 1983 (1983).

[24] J. S. Cohen, J. Phys. B: At. Mol. Phys. 18, 1759 (1985).

[25] C. J. Wood, C. R. Feeler, and R. E. Olson, Phys. Rev. A 56, 3701 (1997).

[26] E. Wigner, Phys. Rev. 40, 749 (1932).

[27] A. E. S. Green, D. L. Sellin, and A. S. Zachor, Phys. Rev. 184, 1 (1969).
[28] R. H. Garvey, C. H. Jackman, and A. E. S. Green, Phys. Rev. A 12, 1144 (1975).

[29] B. Sulik and K. Tökési, Adv. Quantum Chem. 52, 253 (2007).

[30] C. O. Reinhold and R. E. Olson, Phys. Rev. A 39, 3861 (1989).

[31] L. Sarkadi and R. O. Barrachina, Phys. Rev. A 71, 062712 (2005).

[32] C. O. Reinhold and C. A. Falcón, Phys. Rev. A 33, 3859 (1986).

[33] E. Clementi and C. Roetti, At. Data Nucl. Data Tables 14, 177 (1974).

[34] J. N. Silvermann, O. Platas, and F. A. Matsen, J. Chem. Phys. 32, 1402 (1960).

[35] O. Chuluunbaatar, I. V. Puzynin, P. S. Vinitsky, Yu. V. Popov, K. A. Kouzakov, and C. Dal Cappello, Phys. Rev. A 74, 014703 (2006).

[36] P. Khan, Sh. K. Datta, D. Bhattacharyya, and A. S. Ghosh, Phys. Rev. A 29, 3129 (1984).

[37] K. Pachucki and J. Sapirstein, Phys. Rev. A 63, 012504 (2000).

[38] C. L. Kirschbaum and L. Wilets, Phys. Rev. A 21, 834 (1980).

[39] A. B. Voitkiv, B. Najjari, and J. Ullrich, J. Phys. B: At. Mol. Phys. Opt. 36, 2591 (2003).

[40] D. H. Madison, D. Fischer, M. Foster, M. Schulz, R. Moshammer, S. Jones, and J. Ullrich, Phys. Rev. Lett. 91, 253201 (2003).

[41] M. Foster, D. H. Madison, J. L. Peacher, M. Schulz, S. Jones, D. Fischer, R. Moshammer, and J. Ullrich, J. Phys. B: At., Mol., Phys. Opt. 37, 1565 (2004).

[42] J. Fiol, S. Otranto, and R. E. Olson, J. Phys. B: At., Mol., Phys. Opt. 39, L285 (2006).

[43] M. F. Ciappina and W. R. Cravero, J. Phys. B: At., Mol., Opt. Phys. 39, 1091 (2006).

[44] A. L. Harris, D. H. Madison, J. L. Peacher, M. Foster, K. Bartschat, and H. P. Saha, Phys. Rev. A 75, 032718 (2007).

[45] F. Járai-Szabó and L. Nagy, J. Phys. B: At., Mol., Opt. Phys. 40, 4259 (2007).

[46] F. Járai-Szabó and L. Nagy, Nucl. Instrum. Methods, Phys. Res. B 267, 292 (2009).

[47] M. McGovern, C. T. Whelan, and H. R. J. Walters, Phys. Rev. A 82, 032702 (2010).

[48] M. McGovern, D. Assafrão, J. R. Mohallem, C. T. Whelan, and H. R. J. Walters, Phys. Rev. A 81, 042704 (2010).

[49] J. Colgan, M. S. Pindzola, F. Robicheaux, and M. F. Ciappina, J. Phys. B: At., Mol., Opt. Phys. 44, 175205 (2011).

[50] K. A. Kouzakov, S. A. Zaytsev, Y. V. Popov, and M. Takahashi, Phys. Rev. A 86, 032710 (2012).

[51] F. Járai-Szabó and L. Nagy, Eur. Phys. J. D 69, 4 (2015).

[52] F. Navarrete, M. F. Ciappina, L. Sarkadi, and R. O. Barrachina, Nucl. Instrum. Methods, Phys. Res. B 408, 165 (2017).

[53] M. Schulz, Adv. At., Mol., Opt. Phys. 66, 507 (2017).

[54] K. N. Egodapitiya, S. Sharma, A. Hasan, A. C. Laforge, D. H. Madison, R. Moshammer, and M. Schulz, Phys. Rev. Lett. 106, 153202 (2011).

[55] R. D. Rivarola and P. D. Fainstein, Nucl. Instrum. Methods, Phys. Res. B 205, 448 (2003).

[56] D. V. Karlovets, G. L. Kotkin, and V. G. Serbo, Phys. Rev. A 92 , 052703 (2015).

[57] L. Sarkadi, I. Fabre, F. Navarrete, and R. O. Barrachina, Phys. Rev. A 93, 032702 (2016).

[58] F. F. Komarov and M. M. Temkin, J. Phys. B: At. Mol. Phys. 9, L255 (1976). 